\title{
ON SOME COMPOSITIONS OF HADAMARD TYPE IN CLASSES OF ANALYTIC FUNCTIONS
}

\author{
BY C. LOEWNER AND E. NETANYAHU ${ }^{1}$
}

Communicated by I. J. Schoenberg, May 22, 1959

1. Introduction. In a recent paper by G. Polya and I. J. Schoenberg [1] the following conjecture ascribed to Mandelbrojt-Schiffer was stated:

CONJECTURE I. Let $S$ be the class of regular, and schlicht functions in the unit circle.

Let

$$
f(z)=\sum_{n=1}^{\infty} a_{n} z^{n},\left(a_{1}=1\right)
$$

and

$$
g(z)=\sum_{n=1}^{\infty} b_{n} z^{n},\left(b_{1}=1\right)
$$

be the representing power series of two elements of $S$. Then the composition

$$
h(z)=\sum_{n=1}^{\infty} \frac{a_{n} b_{n}}{n} z^{n}
$$

leads to an element of the same class.

A little weaker conjecture than I would be the following:

CONJECTURE II. $h(z)$ has a nonvanishing derivative in $|z|<1$.

The Conjecture I would mean that under this composition rule $S$ forms a semi-group containing the unit element $z /(1-z)^{2}$.

The interest in Conjectures I and II lies in the fact that even from the weaker Conjecture II Bieberbach's conjecture would follow immediately.

Indeed by setting

$$
g(z)=z+\frac{1}{n} z^{n}
$$

one would obtain the composed function

${ }^{1}$ This paper was written by the first author under research contract of the $\mathrm{Na}$ tional Science Foundation, N.S.F. 03020, and the work of the second author was sponsored in part by the Office of Scientific Research of the ARDC of U. S. Air Force, through its European Office, under Contract No. Af 61(052)-187. 


$$
h(z)=a_{1} z+\frac{a_{n}}{n} z^{n}
$$

and from the nonvanishing of its derivative in $|z|<1$ the inequality

$$
\left|\frac{a_{n}}{a_{1}}\right| \leq n
$$

would follow.

We shall prove that the even weaker Conjecture II is false. The possibility still remains that the conjectures are true for functions with real coefficients.

Another disproof of Conjecture II by B. Epstein and I. J. Schoenberg was kindly communicated to the authors by I. J. Schoenberg.

2. Disproof of Conjecture II. We consider all functions mapping the unit circle onto the plane slit along an arbitrary half line mapping $z=1$ into $\infty$.

They have after the usual normalization $f(0)=0, f^{\prime}(0)=1$ the following form

$$
f_{\epsilon}(z)=\int_{0}^{z} \frac{1+\epsilon t}{(1-t)^{3}} d t=z+b_{2} z^{2}+\cdots
$$

with

$$
b_{n}=n \epsilon+\frac{1-\epsilon}{2}(n+1), \quad(\epsilon \text { constant },|\epsilon|=1)
$$

We compose now two functions of type (2.1) according to composition rule (1) and differentiate. The power series representing the derivative has coefficients of $z^{n-1}$ of the form

$$
\begin{aligned}
& b_{n} c_{n}=\left[n \epsilon+2^{-1}(1-\epsilon)(n+1)\right]\left[n \eta+2^{-1}(1-\eta)(n+1)\right], \\
& \qquad|\epsilon|=|\eta|=1 .
\end{aligned}
$$

Summing up, we obtain the function

$$
\frac{1}{4(1-z)^{3}}\left[(1-\epsilon)(1-\eta) z^{2}-(3(1-\epsilon)(1-\eta)-4 \epsilon \eta) z+4\right]
$$

We will show that not for all pairs $\epsilon, \eta$ of absolute value 1 , the zeroes of

$(2.3)(1-\epsilon)(1-\eta) z^{2}-[3(1-\epsilon)(1-\eta)-4 \epsilon \eta] z+4=0$

are outside the unit circle. 
Take $\epsilon,|\epsilon|=1$, arbitrary but different from \pm 1 , and vary $\eta$ near $\eta=+1$. If $\eta=1$, the equation (2.3) reduces to a linear equation with the root $z=-\bar{\epsilon}$. If $\eta$ is close to 1 we write $\eta=e^{i \theta}$ and call the root close to $z=-\bar{\epsilon}$, corresponding to a small $\theta, z(\theta)$. We now compute $d z / d \theta$ at $\theta=0$. We obtain by differentiating (2.3) after substitution of $z=z(\theta)$ and $\eta=e^{i \theta}$ and subsequent substitution $\theta=0$

$$
\left(\frac{d z}{d \theta}\right)_{\theta=0}=i \frac{(\epsilon+1)^{2}}{4 \epsilon^{3}} .
$$

Hence

$$
\left(\frac{1}{z} \frac{d z}{d \theta}\right)_{\theta=0}=-i \frac{\left(\epsilon^{1 / 2}+\epsilon^{-1 / 2}\right)^{2}}{4 \epsilon} .
$$

The last expression is not purely imaginary if $\epsilon \neq \pm \pm 1$. Hence the contradiction.

We should like to remark that one can also obtain another disproof of Conjecture I by composing $f_{\epsilon}(z)$ with itself and check the well known inequality $\left|a_{2}^{2}-a_{3}\right| \leqq 1$ for schlicht mappings. One easily computes that this inequality is not satisfied for instance for $\epsilon=i$.

However, our previous consideration is preferable because it even disproves II and is of absolutely elementary nature.

\section{REFERENCE}

1. G. Polya and I. J. Schoenberg, Remarks on de la Vallée Poussin means and convex conformal maps of the circle, Pacific J. Math. vol. 8 no. 2 pp. 295-335.

STANFORd UNIVERSITY AND

Technion, Israel Institute of Technology, Haifa 